\title{
Cervical exenteration
}

\section{Uma M. Sachdeva, Michael Lanuti}

Division of Thoracic Surgery, Massachusetts General Hospital, Boston, MA, USA

Correspondence to: Michael Lanuti, MD. Division of Thoracic Surgery, Massachusetts General Hospital, 55 Fruit Street, Founders 7, Boston, MA 02114. USA. Email: mlanuti@mgh.harvard.edu.

\begin{abstract}
Cervical exenteration is a radical procedure for the treatment of locally invasive cancers of the trachea, esophagus, or thyroid, as well as recurrent tumors at the site of a tracheal stoma, and occasionally for benign disease. Exenteration involves removal of the larynx, pharynx, esophagus, and trachea, as well as associated lymphatic tissue. The tracheal stump is brought up as a cervical or mediastinal tracheostomy, depending on the length and the location of the distal resection site. The alimentary tract can be reconstructed with several types of conduits, but most commonly the stomach or left colon are used. Tension on the innominate artery must be avoided when repositioning the trachea to prevent innominate artery erosion. Tension on the artery can be addressed by either dividing the vessel or by transposing the trachea inferior and lateral to the innominate artery and vein. Overall, cervical exenteration is associated with a significant risk of morbidity, including anastomotic leak, innominate artery erosion, and tracheostomy dehiscence with subsequent mediastinitis, as well as the potential for postoperative death. Nevertheless, in highly selected patients, it can provide an unparalleled opportunity for either cure or palliation, with functional results equivalent to that of total laryngectomy.
\end{abstract}

Keywords: Mediastinal tracheostomy; conduit; skin flap; aortic homograft; reconstruction

Submitted Nov 28, 2017. Accepted for publication Feb 27, 2018.

doi: 10.21037/acs.2018.03.05

View this article at: http://dx.doi.org/10.21037/acs.2018.03.05

\section{Introduction}

Cervical exenteration involves removal of the pharynx, larynx, and affected portions of the esophagus and suprasternal trachea for curative or palliative intent. While infrequently performed by thoracic surgeons in the current era, the origins of this procedure reside in both the fields of thoracic surgery and laryngeal surgery, with the initial description of the technique for cervicomediastinal exenteration with anterior mediastinal tracheostomy by Hermes Grillo, MD. This radical procedure, while extensive, is in some cases the only option for definitive treatment of primary or secondary malignancies involving the upper aerodigestive tract, and in the best of circumstances can offer an outcome that is the functional equivalent of a laryngectomy. Following removal of the upper airway, including the larynx and cervicomediastinal trachea, and the pharyngoesophageal portion of the alimentary tract, a significant reconstructive effort is required to restore continuity of the digestive tract, fill the cervicomediastinal defect with skin and soft tissue, and construct an end tracheostomy. Depending on the location and length of the residual tracheal stump, the tracheocutaneous anastomosis can be created as a standard cervical tracheostomy or may require creation of an anterior mediastinal tracheostomy, if the entire cervical trachea has been removed as specimen. Given the extent of the dissection, resection, and reconstruction required for this procedure, reported morbidity and mortality is significant. Nevertheless, for survivors, this operation provides good functional outcomes, palliation of obstructive symptoms, and potential for cure. Therefore, great care must be taken in selecting patients who are most likely to benefit from this extensive procedure and have an expected post-operative survival longer than six months.

\section{Indications}

Cervical exenteration may be required for complete 
resection of tumors of the upper aerodigestive tract or thyroid gland that are locally invasive to the surrounding structures of the neck. These tumors include adenoid cystic carcinoma of the trachea; hypopharyngeal, postcricoid, or cervicothoracic esophageal carcinomas invading the upper trachea; differentiated carcinomas of the thyroid that involve the upper trachea, including papillary, follicular, or mixed type carcinoma; poorly differentiated but localized carcinoma of the thyroid involving the upper trachea; and recurrent carcinoma of the larynx that recurs at the site of prior tracheostomy. Some benign conditions may also benefit from this approach, including post-radiation stenosis of the larynx, pharynx, trachea, and esophagus. Resection may be undertaken with curative or palliative intent to relieve obstruction of the upper aerodigestive tract. Alternatively, exenteration may be undertaken as salvage therapy after failure of primary chemoradiation for laryngotracheal or pharyngoesophageal tumors, most often squamous cell carcinoma of the larynx, hypopharynx, or postcricoid esophagus.

\section{Preoperative workup}

Preoperative evaluation should begin with complete clinical staging to determine whether exenteration would provide a survival or palliative benefit. Evaluation should include diagnostic quality CT neck and chest along with positron emission tomography (PET/CT) to evaluate for distant or regional nodal metastases and establish the potential oncologic benefit of extensive resection. Furthermore, the feasibility of a complete resection should be determined by thorough evaluation of the extent of disease and degree of local invasion. Specifically, the presence of direct invasion into the cervical spine must be assessed, as this would preclude an attempt at resection, as well as involvement of the cranial vessels, specifically the carotid and vertebral arteries. The trachea should be fully evaluated by bronchoscopy, and measurements should be undertaken prior to surgery to assess the location and degree of tracheal involvement. The larynx and oropharynx should also be assessed with direct laryngoscopy and oropharyngoscopy. For complete evaluation of the esophagus, barium esophogram and upper GI endoscopy should be completed.

Preoperative workup is also essential for determining the optimal approach for reconstruction. When performed by thoracic surgeons, reconstruction of the esophagus includes either gastric or colon conduits, necessitating preoperative assessment of the vascular supply to both of these organs, as well as endoscopic assessment of their viability and the presence of any intrinsic disease that may preclude their use as a suitable graft. Mesenteric angiogram can be considered to better understand the blood supply of a potential colon conduit. If these organs cannot serve as viable conduits, a myocutaneous pedicle flap from the chest wall (1) or a free flap from the forearm (2) can be transposed and fashioned to create a neoesophagus, an approach that is sometimes favored by laryngeal oncologic surgeons. Jejunal free flap is another alternative conduit that requires vascular anastomosis to neck vessels (3).

Preoperative cerebral angiogram is required if there is potential for division of the innominate artery during creation of an anterior mediastinal tracheostomy, or if carotid artery involvement of the primary tumor is suspected. This will be discussed further below.

Cervical exenteration is a long operation that requires several hours to complete and carries with it high cardiopulmonary risk (4). All patients should therefore undergo preoperative cardiac and pulmonary evaluation including functional testing, if indicated. Baseline neurologic function should be documented, given the potential for intraoperative division of the innominate artery, injury to the cerebral vessels, or direct tumor involvement of the carotid or vertebral arteries. Nutritional status should be optimized to facilitate tolerance of the procedure and to support the extended recovery. Temporary feeding tubes (often jejunal) should be considered upon performing cervical exenteration to allow for adequate post-operative nutrition while the pharyngeal reconstruction heals.

\section{Operative setup}

On the day of procedure, hemodynamic monitoring must be initiated preoperatively. This should include placement of an arterial line, oxygen saturation probe, and at least two large bore intravenous catheters. These lines should be placed in such a way that they would be unaffected by potential division of the innominate artery or the left innominate vein during the procedure. The patient is then brought into the operating room and positioned supine on the operating room table, with at least one arm abducted in the event that upper extremity access is needed during the procedure. Endotracheal intubation should be planned carefully given the location and extent of the tumor. Alternatively, if an existing cervical tracheostomy is present, a reinforced (or armored) endotracheal tube is placed directly through that site. EEG monitoring is required if 


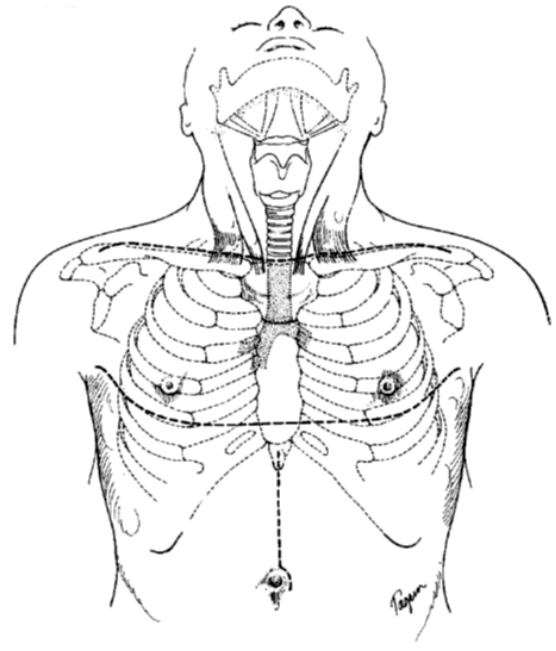

Figure 1 Incisions for cervical exenteration. The superior incision allows access to the neck and mediastinum. The inframammary incision is used to create a bipedicled advancement flap, according to the technique described by Grillo et al. A midline laparotomy allows preparation of the conduit, usually stomach or colon, and placement of a jejunostomy tube for enteral feeding postoperatively. With permission from Grillo and Mathisen, 1990.

there is anticipated division or involvement of the cerebral vessels. The sterile operative field should span from the chin to the pubis, and laterally from mid-axillary line to mid-axillary line. If the need for skin graft is anticipated, the thigh should also be included in the sterile field. If the need for forearm free flap is anticipated, the arm should be similarly prepared.

The initial incision is a low collar incision, oriented transversely above the sternal notch and placed superior to the clavicular heads (Figure 1). The prior tracheostomy site, if present, should be included within the incision. Any skin involved with tumor or with radiation change is excluded from the incision and resected with the underlying specimen. Despite preoperative imaging, the extent of tumor involvement is often difficult to assess accurately prior to surgery. Therefore, the procedure should always begin with a complete neck exploration. After raising skin flaps, the region is thoroughly inspected in all directions. Superiorly, skin and muscle flaps are mobilized to above the hyoid bone. Inferiorly, the sternal notch is exposed. The lateral extent of dissection includes the carotid artery and internal jugular vein. The strap muscles are separated to expose the underlying trachea and esophagus. The prevertebral fascia is examined for any tumor invasion.

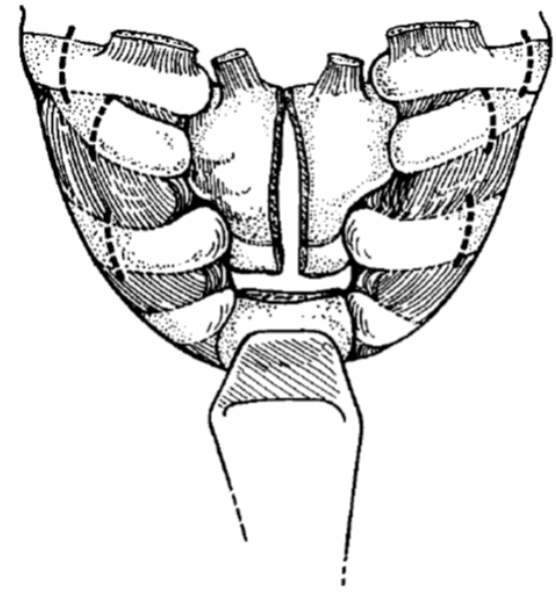

Figure 2 Dissection and resection of the anterior breast plate. The manubrium is split to facilitate mediastinal exploration and dissection. Both clavicular heads are removed along with $4 \mathrm{~cm}$ of the medial clavicles. The first and second costal cartilages are resected, along with the first intercostal muscle. With permission from Grillo and Mathisen, 1990.

If the mediastinum cannot be fully assessed by the collar incision, the incision should be extended laterally over the clavicles and the inferior flap raised over the pectoralis fascia at the midline. The upper sternum is then split vertically from the midline to the level of the second interspace, and then extended transversely across the interspace, to allow for complete mediastinal exploration (Figure 2) (5). This exposed anterior sternal plate can then be resected if anterior mediastinal tracheostomy is required.

\section{Management of the thyroid}

If the thyroid is not involved, the gland can be split in the midline and each lobe can be retracted laterally to expose the underlying trachea. This retraction should allow preservation of both thyroid and parathyroid function. However, if exploration is undertaken for locally invasive thyroid carcinoma, complete resection may be required. Outcomes following cervical exenteration undertaken for thyroid carcinoma are discussed further below.

\section{Division of the trachea and esophagus}

Resection of the trachea begins with identifying the limits of the resection, with the goal of obtaining a roughly 1.5 -centimeter margin of disease-free tissue as part of the 
specimen. Management of the tracheal stump is dependent on residual tracheal length. In cases where the trachea can be divided several centimeters above the sternal notch, a cervical tracheostomy can be created. However, if the trachea must be divided behind the sternum or if the operation is being undertaken for recurrence at a prior tracheostomy site, the patient will require creation of an anterior mediastinal tracheostomy.

At this point, the manubrium should be incised at the midline, and the incision extended transversely over the second intercostal space for several centimeters bilaterally (Figure 2). Myocutaneous flaps are raised and retracted laterally to expose the bony breast plate. Each clavicle is then divided four centimeters from the medial aspect, taking care not to injure the underlying subclavian veins. After releasing the first intercostal muscle from the sternum and ribs, the first and second costal cartilages are divided bilaterally, and the clavicular heads, costal cartilages, and manubrium are excised and removed en bloc. The sternum can be divided in the midline prior to resection of the anterior breast plate to facilitate extraction; this also enhances exposure during the initial exploration. The trachea is then examined and the distal limit of resection is determined, taking into account the need for adequate margin on the specimen distal to the tumor. Lateral traction sutures are then placed on both sides of the trachea, around one cartilaginous ring below the intended level of transection. The trachea is then divided, and the tracheal stump is intubated within the operative field and cross table ventilation initiated. A margin from the tracheal stump should be sent for pathologic analysis from a frozen section to ensure that it is negative for residual tumor.

The esophagus is then evaluated for tumor involvement. If involvement is limited, the esophagus may be preserved or primarily repaired after partial-circumference resection. If the esophagus is extensively involved, the distal extent of resection is determined and the esophagus is circumferentially dissected and then sharply divided. The dissection then proceeds superiorly from the mediastinum to the neck. The field of resection extends from carotid sheath to carotid sheath, and extending posteriorly to the prevertebral fascia. If necessary, one internal jugular vein may be sacrificed. One carotid artery may also be taken if needed, with arterial reconstruction or bypass to restore cerebral perfusion. Once the level of the larynx has been reached, the suprahyoid muscles, including the mylohyoid, geniohyoid, and genioglossus muscles, the hyoid bone, and the muscles of the larynx are transected. With this, both superior laryngeal nerves and vessels are taken with the specimen. As the dissection continues posteriorly, the pharynx is divided and the epiglottis included with the resection. At this point the specimen is extracted with larynx, pharynx, trachea, esophagus, and nodal tissue en bloc. The superior resection margin is sent for frozen section analysis.

\section{Reconstruction}

Reconstruction begins with the esophagus. Conduits for reconstruction include stomach, colon, or microvascular free flaps of the jejunum (3), radial forearm (2), or pectoralis (6). Free flaps are more commonly used for tumors arising above the thoracic inlet. For tumors at or below the thoracic inlet, gastric or colonic transposition is favored. The organ for transposition is prepared within the abdomen. The colon is mobilized from the hepatic flexure to the sigmoid colon, and a left colon conduit is created on a pedicle arising from the left colic artery. Alternatively, if the left colon is unable to be used, right colon can be substituted with a somewhat less reliable result $(5,7)$. A substernal tunnel is made and the vascular pedicle is brought behind the stomach. The distal end of the conduit is anastomosed to the anterior wall of the stomach, and the proximal end of the conduit is anastomosed to the pharynx (base of tongue) using two layers of 4-0 interrupted silk or vicryl sutures. This anastomosis can be performed in either an end-toend or end-to-side fashion, with the blind end of the colon oversewn (Figure 3). A pedicled omental flap based on the right gastroepiploic artery is also prepared by separating it from the colon and stomach. This flap is brought up retrosternally and divided into two tongues, taking care not to disrupt the blood supply. One tongue is used to wrap the anastomosis as a buttress to decrease the chance of anastomotic leak; the other tongue is later used to support the tracheal stoma (Figure 4). If a gastric conduit is used, the omentum is left attached to the greater curvature of the stomach, and the stomach and omentum are brought into the mediastinum as one unit. The proximal anastomosis of any pharyngeal reconstruction is at risk for leak since many of these patients were previously treated with radiation, where tissue integrity and wound healing is not ideal. It is imperative that the proximal anastomosis be covered or reinforced with viable tissue (i.e., omentum along with a pedicled myocutaneous pectoralis flap to make up for loss of tissue domain).

If cervical tracheostomy is unable to be performed, 


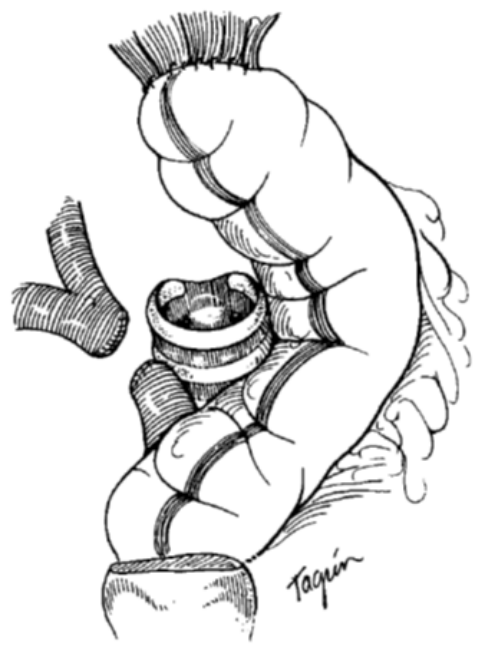

Figure 3 Esophageal reconstruction. The conduit (here, colonic) is brought into the mediastinum to the left of the tracheal stump and anastomosed to the base of the tongue in two layers. With permission from Grillo and Mathisen, 1990.
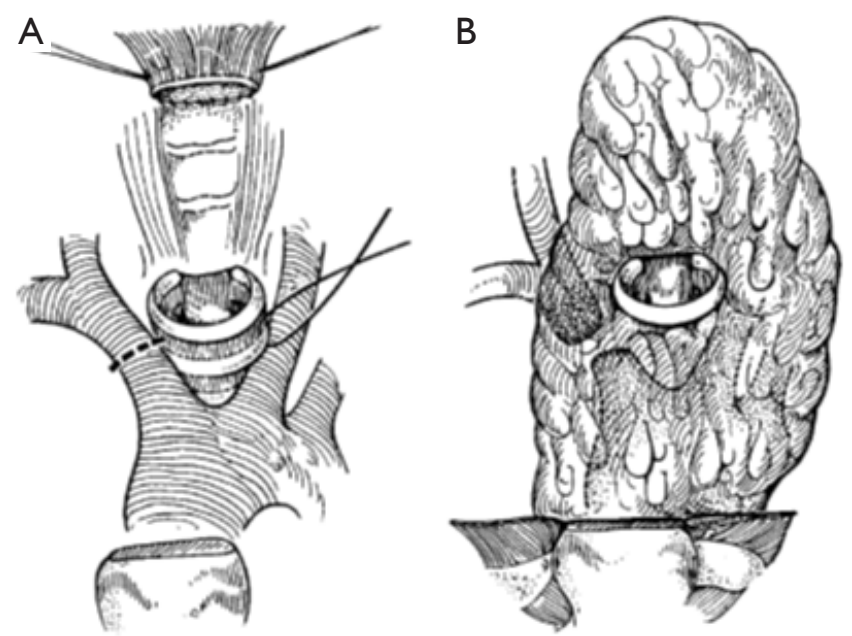

Figure 4 Tracheal resection. The diseased portion of the trachea is resected, and the innominate artery is divided (dashed line) if tension exists between the artery and the distal tracheal stump (A). Prior to maturing the tracheostomy, the tracheal stump is wrapped with omentum to protect the innominate artery from potential erosion (B). With permission from Grillo and Mathisen, 1990.

creation of an anterior mediastinal tracheostomy is required. The tracheal stoma remains to the right of the gastric or colonic conduit. The cut end of the trachea is brought out of the mediastinum with aid of the lateral traction sutures. Proximity of the tracheal stoma to the

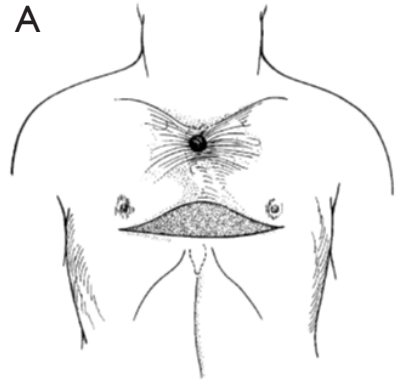

B

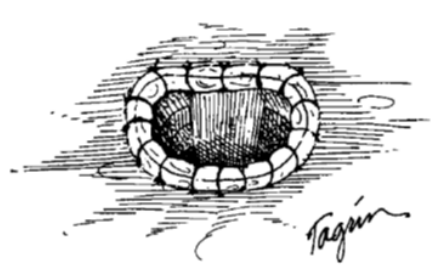

Figure 5 Creation of the anterior mediastinal tracheostomy using a bipedicled skin flap. The tracheostomy is brought out through the flap below the cervical incision site (A). The tracheal stump is sutured to the skin flap with interrupted vicryl sutures. With permission from Grillo and Mathisen, 1990.

innominate artery must be carefully assessed to prevent erosion of the innominate artery, a grave complication that, when occurs, results in death. Initial description of the cervical exenteration procedure by Grillo and colleagues describes a technique of elective ligation of the innominate artery in this circumstance, with oversewing of the proximal and distal ends (5). Prior to elective ligation, the artery is clamped and the EEG observed for changes over 20 minutes, with the artery divided only if no changes are observed. This division must occur proximal to the carotidsubclavian bifurcation. The stoma is then wrapped in omentum both to separate it from the innominate artery and to protect against potential dehiscence (Figure 4) $(5,8)$. The tracheostomy is brought out through a bipedicled anterior cutaneous flap (Figure 5). To create this flap, a second incision is made beneath both inframammary folds, extending between the anterior axillary lines. A cutaneous flap is created by dissecting anterior to the pectoral fascia and then mobilizing the flap superiorly, into the mediastinal defect. The stoma is then brought through an opening in the flap and sutured to the skin using 4-0 vicryl sutures. Multiple flat Penrose drains are then placed bilaterally in the neck, mediastinum, and subcutaneous spaces. The upper incision is closed primarily in layers with sutures. The lower incision requires coverage with a split thickness skin graft that is harvested from the anterior thigh to prevent placing tension on the flap or on the mediastinal tracheostomy that would promote flap failure or stomal dehiscence.

Orringer has described an alternative technique by which division of the innominate artery and reconstruction with bipedicled skin flap was avoided by mobilizing the distal tracheal stump to a location inferior to the 


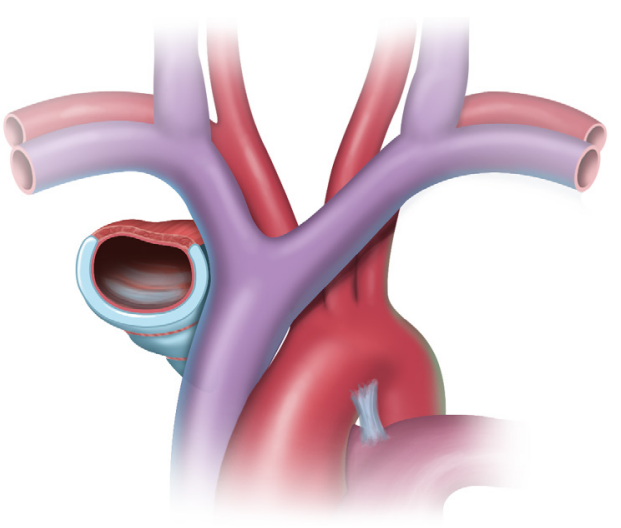

Figure 6 Transposition of the tracheal stump. To avoid tension between the tracheal stump and the innominate artery, the trachea can be transposed laterally and inferior to the innominate vessels. Modified with permission from Orringer, 1992.

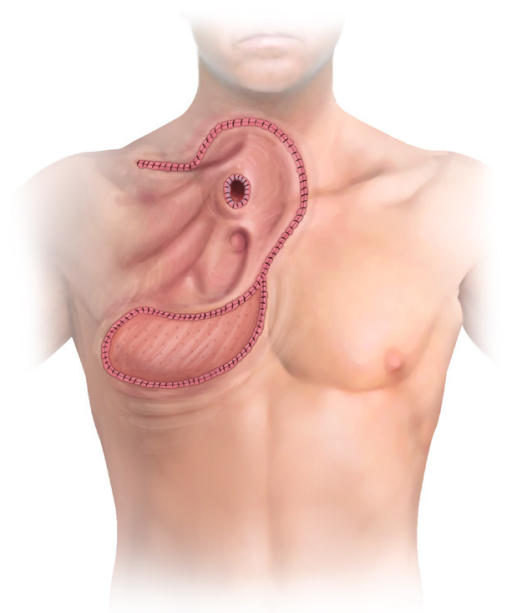

Figure 7 Thoracoacromial skin flap. This flap can be used when the tracheal stump has been repositioned below and laterally to the innominate vessels. The inframammary wound is covered with a split thickness skin graft. Modified with permission from Orringer, 1992.

innominate artery (9). Using this approach, tension on the tracheocutaneous anastomosis is avoided by developing a space beneath and to the right of the innominate artery and vein. The tracheal stump is then transposed to a position beneath the innominate artery, allowing an additional 1.5 to 2 centimeters of length to be brought out of the mediastinum (Figure 6). As such, the mediastinal defect can be reconstructed and the stoma created through a thoracoacromial or nipple flap, if needed (Figure 7). This flap is created by mobilizing the skin and subcutaneous tissue off the underlying pectoralis muscle and rotating it superiorly and medially to fill the mediastinal and cervical defect. The tracheostomy is then created through this flap. As with Grillo's technique, the inferior defect created by mobilization of this flap is reconstructed with a split thickness skin graft that is harvested from the anterior thigh or lower abdomen. Initial description of this approach indicated the necessity of a distal tracheal stump of at least 5 centimeters (9), though more recent reports have reported success with a shorter distal stump (10).

\section{Postoperative care}

Post-operatively, patients should recover in an intensive care unit, where their airway and hemodynamics can be closely monitored. If mechanical ventilation is required, as is often the case, this is accomplished through a wirereinforced endotracheal tube placed through the tracheal stoma, or through a customized shortened tracheostomy tube. The tracheal balloon should not be overinflated to avoid trauma to the remaining trachea, and the tip of the tube must end above the carina. Patients are kept on humidified air once mechanical ventilation is no longer needed. Nasogastric tube remains in place for at least seven days to prevent regurgitation or flow of gastric secretions out of the mouth and into the mediastinal tracheostomy. The nasogastric tube may be removed once the patient is ambulating and is able to sit upright while coughing. Calcium levels are checked postoperatively in the event of iatrogenic hypoparathyroidism, and calcium and vitamin D supplementation are given as needed. Most patients undergo jejunostomy tube placement for enteral access at the time of the operation, which is used for feeding and medications during the postoperative period.

\section{Complications}

Despite the relative infrequence of this procedure, there are several case series reporting the results of cervical exenteration with or without anterior mediastinal tracheostomy that highlight the complications associated with this procedure. Orringer reported his results of 44 patients with anterior mediastinal tracheostomies, 34 of whom underwent cervical exenteration for laryngeal, thyroid, or cervical esophageal cancers (9). Fourteen of these patients underwent transposition of the tracheal stump below and to the right of the innominate artery. Six patients (18\%) undergoing cervical exenteration 
died postoperatively. One death was due to innominate artery erosion, one to cerebrovascular accident, one to acute myocardial infarction, one to sepsis resulting from pharyngogastric anastomotic leak, one to respiratory and renal complications, and one to hepatorenal syndrome. Of the surviving 28 patients, only 9 (32\%) had an uncomplicated hospital course, emphasizing the high morbidity associated with this procedure. Major complications included six leaks from the pharyngeal anastomosis, nine cases of iatrogenic hypoparathyroidism and hypothyroidism, three cerebrovascular accidents, three cases of cervical chyle fistula, one case of delayed splenic rupture, and one case of hepatic failure caused by cirrhosis. Average hospital stay was 26.2 days, with a range from 10 to 51 days. Four patients (14\%) were discharged within two weeks of the operation; $75 \%$ were discharged between three and eight weeks postoperatively. Conduit complications included necrosis of the upper end of a gastric conduit, which was addressed with a free jejunal interposition graft, and venous infarction of a colonic graft, which was addressed with gastric interposition. Chyle leaks required reoperation and ligation of the cervical thoracic duct in two out of three patients. Neurologic symptoms resulting from cerebrovascular accidents were transient in two out of three patients with this complication and resulted from ligation and resection of the common carotid artery.

An earlier study by Grillo and Mathisen reported similar high morbidity associated with this operation (5). Eighteen patients underwent cervical exenteration for carcinoma of the cervical esophagus, carcinoma of the thyroid, adenoid cystic carcinoma, recurrent laryngeal cancer, or complications from prior radiation. Average hospital stay was 34.2 days, with the range from 15 to 58 days. The innominate artery was electively divided in seven patients. Ten patients were reconstructed with colon, and four were reconstructed with stomach, while the remainder underwent primary repair of the esophageal defect. Ten patients $(56 \%)$ had no major complications reported, while eight patients (44\%) experienced major complications. Two leaks were reported, one from a primary esophageal repair, and the other from a pharyngocolonic anastomosis using the right colon as a conduit. Two patients experienced tracheal stomal dehiscence, one of which was managed with debridement and was complicated by later stenosis requiring revision. The second patient experienced complete stomal separation from the bipedicled skin flap used for mediastinal reconstruction, and required rotation of a pectoralis muscle flap to fill the defect and protect the underlying vascular structures. One patient experienced right sided hemiplegia after resection of the bifurcation of the innominate artery, which improved after bypass from the left subclavian to the right common carotid artery. One patient experienced obstruction of the colonic graft within the substernal mediastinal tunnel, requiring surgical revision. Four patients required prolonged mechanical ventilation, three of whom had pneumonia. Wound dehiscence requiring operative sternal debridement was observed in one patient. One patient experienced iliofemoral thrombosis. There was also one perioperative death, due to sepsis resulting from ischemic necrosis of a reversed gastric tube used for reconstruction of the alimentary tract.

A more recent report from Conti et al. describes six patients who underwent cervical exenteration with anterior mediastinal tracheostomy (11). Two of these were performed for esophageal squamous cell carcinoma, one for subglottic squamous cell carcinoma, two for recurrence at a prior tracheal stoma, and one for recurrence at the anastomotic site of a previous tracheal resection. Reconstruction of the defect and stoma creation were performed using a pectoralis major myocutaneous island flap based off the thoracoacromial vessels. Five patients underwent pharyngogastric anastomosis, and one patient was reconstructed with a left colonic interposition graft. Four $(67 \%)$ of these patients experienced a major postoperative complication, and there was one postoperative death. Two patients experienced anastomotic leaks, one patient developed necrosis of the myocutaneous flap, and one patient developed a pulmonary embolus. In the case of flap failure, the left innominate vein had been ligated intraoperatively, and postoperative venous congestion of the flap resulted in necrosis, stomal disruption, and rupture of the innominate artery, resulting in death. Both cases of anastomotic leak were addressed surgically by rotating the contralateral pectoralis muscle to close the defect.

One additional case report by Trachiotis and Hix described the development of a tracheogastric fistula following cervical exenteration for esophageal squamous cell carcinoma (12). The esophagectomy had been complicated by a tear of the membranous wall of the trachea that extended to the proximal right mainstem bronchus. This was repaired primarily using a right thoracotomy approach. Reconstruction of the alimentary tract was then performed with pharyngogastric anastomosis. On the ninth postoperative day, a tracheogastric fistula was identified between the lesser curvature of the stomach and the lateral posterior wall of the tracheostomy. This was managed 
initially with drainage, followed by definitive surgical repair by mobilization of the strap muscles and placement of these muscles as an interposition flap. The patient subsequently developed a second tracheogastric fistula located more distally several months later, which was repaired surgically using a median sternotomy approach.

Grillo et al. also reviewed their experience managing patients with locally invasive thyroid carcinoma involving the airway (13). In this cohort, seven out of 52 patients required cervicomediastinal exenteration with or without mediastinal tracheostomy. Three of these patients underwent esophagectomy with reconstruction via colonic interposition graft. Three patients underwent partial noncircumferential resection of the esophagus with primary esophageal repair. Along with cervical exenteration, bilateral modified neck dissection was performed in three patients, and left posterior triangle dissection was performed in one patient due to the presence of lymph node metastases. One patient required resection of the innominate artery and the carotid-subclavian bifurcation. There were no EEG changes observed at the time of resection, but delayed onset of neurologic symptoms required subsequent carotid reconstruction. One patient underwent prophylactic division of the innominate artery. Six patients required mediastinal tracheostomy, while one patient was able to undergo low cervical tracheostomy. The omentum was mobilized and brought into the neck in four patients. There was one observed post-operative death, with the remaining survival dependent on progression of the underlying disease process. An updated report from this group examining patients who underwent resection for thyroid carcinoma between the years 1964 and 2005 included no additional cases treated with cervical exenteration (14), highlighting the rarity of this procedure in the current era.

\section{Outcomes}

In the best scenario, long term functional outcomes have been reported to be equivalent to those following total laryngectomy. All patients who survived through the initial postoperative period were able to maintain their nutritional status with oral intake $(5,9)$. While long term patency of the alimentary tract was able to be maintained, Grillo and Mathisen report that esophageal dilation was required in six patients. Tracheal stoma revision was required in five patients. Orringer reported initial frequent complaints of postprandial regurgitation with cough, bending, or supine positioning, which improved by 6 months with behavioral modification. Some patients also reported postprandial diarrhea, which improved with diet modification and antispasmodics. Most patients are able to speak via use of an electrolarynx or to achieve functional esophageal or gastric speech.

In all reports, long term survival was dependent on the underlying disease process. Resection can be curative or palliative. In the cases of palliation, most patients die of recurrent or metastatic disease, or their related complications $(5,9)$.

\section{New frontiers}

While esophageal reconstruction has most commonly been described with colon or gastric interposition graft, recent studies have described the use of cryopreserved aortic homograft as a prosthesis to reconstruct defects in the aerodigestive tract. This technique has been reported as a viable technique for reconstructing the larynx after transcervical partial laryngectomy to avoid total laryngectomy (15). In this study, at least $40 \%$ of the cricoid circumference was resected in eight out of 15 patients, with repair using aortic homograft as an extended patch graft, which was sewn in place with multiple interrupted vicryl sutures. There were no major complications reported, and post-operatively, all patients had laryngeal phonation. 14 out of 15 patients were able to resume oral intake. Cryopreserved aortic homograft was thus shown to be a safe and viable reconstructive option following oncologic resection of the upper airway. Following this study, aortic homograft has been used in select cases to reconstruct partial defects in the esophageal wall that are not amenable to primary repair (Figure 8). However, the long-term results of this technique are as yet unknown.

\section{Conclusions}

Cervical exenteration is a radical procedure to remove the affected tissues of the neck and mediastinum and reconstruct the aerodigestive tract for the treatment or palliation of symptoms resulting from locally invasive malignancies of the trachea, esophagus, or thyroid, or benign stricturing of the region following radiation treatment. Potential complications of the procedure are significant, including anastomotic leak, tracheal stoma dehiscence, erosion of the innominate artery, and flap necrosis, all of which can result in death. However, by removing all affected organs and surrounding nodal tissue, this procedure may provide the 

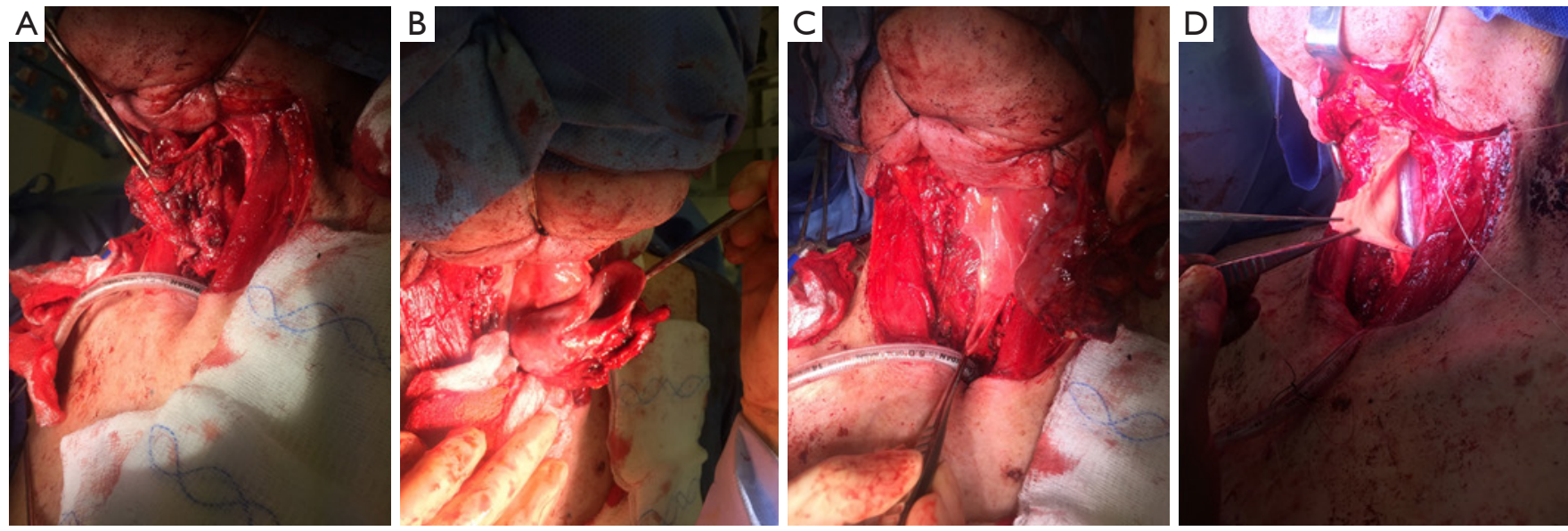

Figure 8 Cervical resection with esophageal reconstruction using aortic homograft. Anterior (A) and posterior (B) views of a resection specimen including the larynx and cervical trachea and the anterior esophagus. The anterior esophageal defect included over $50 \%$ of the circumference (C). This esophageal defect was reconstructed with cryopreserved aortic homograft sewn in place over a silicone tube (D).

only opportunity for cure for locally invasive or recurrent malignancies of the upper airway and cervical esophagus. Careful patient selection is critical to achieving successful outcomes, but in the right patient this approach can afford prolonged survival and functional outcomes equivalent to that of total laryngectomy.

\section{Acknowledgements}

We would like to acknowledge Douglas J. Mathisen, MD and the late Emeritus Professor Hermes Grillo, MD for their valuable contributions to the evolution of this complex surgical modality.

\section{Footnote}

Conflicts of Interest: The authors have no conflicts of interest to declare.

\section{References}

1. LoGiudice JA, Wyler von Ballmoos MC, Gasparri MG, et al. When the Gastrointestinal Conduit for Total Esophageal Reconstruction Is Not an Option: Review of the Role of Skin Flaps and Report of Salvage With a Single-Stage Tubed Anterolateral Thigh Flap. Ann Plast Surg 2016;76:463-7.

2. Varvares MA, Cheney ML, Gliklich RE, et al. Use of the radial forearm fasciocutaneous free flap and montgomery salivary bypass tube for pharyngoesophageal reconstruction. Head Neck 2000;22:463-8.

3. Gaur P, Blackmon SH. Jejunal graft conduits after esophagectomy. J Thorac Dis 2014;6 Suppl 3:S333-40.

4. Fleisher LA, Fleischmann KE, Auerbach AD, et al. 2014 ACC/AHA guideline on perioperative cardiovascular evaluation and management of patients undergoing noncardiac surgery: a report of the American College of Cardiology/American Heart Association Task Force on practice guidelines. J Am Coll Cardiol 2014;64:e77-137.

5. Grillo HC, Mathisen DJ. Cervical exenteration. Ann Thorac Surg 1990;49:401-8; discussion 408-9.

6. Fabian RL. Pectoralis major myocutaneous flap reconstruction of the laryngopharynx and cervical esophagus. Laryngoscope 1988;98:1227-31.

7. Wain JC, Wright CD, Kuo EY, et al. Long-segment colon interposition for acquired esophageal disease. Ann Thorac Surg 1999;67:313-7; discussion 317-8.

8. Kuwabara Y, Sato A, Mitani M, et al. Use of omentum for mediastinal tracheostomy after total laryngoesophagectomy. Ann Thorac Surg 2001;71:409-13.

9. Orringer MB. Anterior mediastinal tracheostomy with and without cervical exenteration. Ann Thorac Surg 1992;54:628-36; discussion 636-7.

10. Gomez-Caro A, Gimferrer JM, Macchiarini P. Technique to avoid innominate artery ligation and perform an anterior mediastinal tracheostomy for residual trachea of less than $5 \mathrm{~cm}$. Ann Thorac Surg 2007;84:1777-9.

11. Conti M, Benhamed L, Mortuaire G, et al. Indications 
and results of anterior mediastinal tracheostomy for malignancies. Ann Thorac Surg 2010;89:1588-95.

12. Trachiotis GD, Hix WR. Repair of tracheogastric fistula after cervical exenteration. Ann Thorac Surg 1996;61:719-21.

13. Grillo HC, Suen HC, Mathisen DJ, et al. Resectional management of thyroid carcinoma invading the airway.

Cite this article as: Sachdeva UM, Lanuti M. Cervical exenteration. Ann Cardiothorac Surg 2018;7(2):217-226. doi: 10.21037/acs.2018.03.05
Ann Thorac Surg 1992;54:3-9; discussion 9-10.

14. Gaissert HA, Honings J, Grillo HC, et al. Segmental laryngotracheal and tracheal resection for invasive thyroid carcinoma. Ann Thorac Surg 2007;83:1952-9.

15. Zeitels SM, Wain JC, Barbu AM, et al. Aortic homograft reconstruction of partial laryngectomy defects: a new technique. Ann Otol Rhinol Laryngol 2012;121:301-6. 